\title{
Asymptotic behaviour for the Vlasov-Poisson System in the stellar dynamics case
}

\author{
Jean Dolbeault \\ Ceremade (UMR CNRS no. 7534), Université Paris IX-Dauphine, \\ Place de Lattre de Tassigny, 75775 Paris Cédex 16, France \\ E-mail: dolbeaul@ceremade.dauphine.fr \\ Óscar Sánchez \& Juan Soler \\ Departamento de Matemática Aplicada, \\ Facultad de ciencias, Universidad de Granada, \\ 18071 Granada, Spain \\ E-mail: ossanche@ugr.es, jsoler@ugr.es
}

May 27, 2003

\begin{abstract}
We study an optimal inequality which relates potential and kinetic energies in an appropriate framework for bounded solutions of the Vlasov-Poisson (VP) system. Optimal distribution functions, which are completely characterized, minimize the total energy. From this variational approach, we deduce bounds for the kinetic and potential energies in terms of conserved quantities (mass and total energy) of the solutions of the VP system and a nonlinear stability result. Then we apply our estimates to the study of the large time asymptotics and observe two different regimes.

Keywords. Vlasov-Poisson system - stellar dynamics - polytropic gas spheres - gravitation - mass - energy - kinetic energy - potential energy - interpolation - Hardy-Littlewood-Sobolev inequality - optimal constant - symmetric nonincreasing rearrangements - Riesz' theorem - bounded solutions - direct variational methods - minimization - scalings - solutions with compact support - Galilean invariance - pseudo-conformal law - variance - dispersion
\end{abstract}

AMS classification (2000). Primary: 35A15, 35B40, 82B40, 82C40; Secondary: 35B35, 35B45, 35J20, 35J60, 76P05, 82D99

\section{Introduction}

The three-dimensional Vlasov-Poisson system can be written in terms of a nonnegative distribution function $f:(0, \infty) \times \mathbb{R}^{3} \times \mathbb{R}^{3} \longrightarrow \mathbb{R}^{+} \cup\{0\}$ and the corresponding mass density $\rho(x, t):=\int_{\mathbb{R}^{3}} f(t, x, v) d v$ as follows:

$$
\left\{\begin{array}{l}
\partial_{t} f+v \cdot \nabla_{x} f-\nabla_{x} \phi \cdot \nabla_{v} f=0, \\
f(t=0, x, v)=f_{0}(x, v), \\
\Delta_{x} \phi=4 \pi \gamma \rho, \quad \lim _{|x| \rightarrow \infty} \phi(t, x)=0,
\end{array}\right.
$$


where $\gamma$ is \pm 1 depending on the physical system under consideration: $\gamma=+1$ corresponds to the evolution of particles subject to their own gravity (gravitational case) and $\gamma=-1$ describes the dynamics of charged particles (plasma physical case).

The purpose of this work is to study the asymptotic behaviour of the solutions of the VP system in the gravitational case by the mean of an associated variational problem.

We first obtain optimal upper and lower bounds of the kinetic and potential energies in terms of the mass and of the minimum of the total energy functional (Theorem 1). These estimates are optimal in the sense that they coincide in the case of one of the socalled polytropic gas spheres solutions (Theorem 2; see [3] for a study of these solutions by means of the associated characteristics system). We reduce the problem to the proof that the minimum of the energy is realized in a class of bounded functions (see below). In Section 3, we completely characterize the minimizers, which in turn gives an optimal constant for an interesting inequality (see Appendix B), and also proves a nonlinear stability result (Theorem 3).

The dispersive character of the solutions of the VP system in the plasma physical case has been proved by using $L^{p}$-estimates of the mass density. As was pointed out in [9], a different qualitative behaviour can be expected for the gravitational case, due to the existence of stationary solutions. Section 4 of this paper is devoted to the study of this case. In terms of the orientation of an inequality which relates the value of the energy, the mass and the momentum of the initial data, we distinguish two situations: either we can derive positive lower bounds for the potential and a norm of the mass density, or we prove that the variance of the density function is of order $t^{2}$ as in the plasma physics case. For that purpose, we extensively use the Galilean invariance of the VP system, and also the pseudo-conformal law as in the dispersive case.

There is a general interest in understanding the large time behaviour of time dependent solutions of the VP system, which has given rise to various approaches in the literature, ranging from the study of the stability of certain solutions $[1,6,7]$ to the analysis of the time evolution of integral quantities (moments, $\mathrm{L}^{p}$-norms, ...), see e.g. [2]. Our dispersion results extend the estimates of J. Batt in [2] to the non spherically symmetric case.

The solutions corresponding to polytropic gas spheres are radial and take the special form

$$
f(x, v)=\left(E_{0}-|v|^{2} / 2-\phi(x)\right)_{+}^{\mu}|x \times v|^{2 k}
$$

(see $[3,4,6]$ for details). In [6] (see $[7,16]$ for more recent results) some of these solutions (the ones corresponding to $0<\mu<\frac{3}{2}+k, k>-1$ ) were obtained as minima of a so-called Energy-Casimir functional. Here, we extend these results and the compactness arguments to the limit case which formally corresponds to $\mu=0$ and $k=0$. Considerations on the total energy functional are fruitless at a first sight, since this functional is not bounded from below in the functional spaces proposed in [6]. This motivates an extra restriction (a uniform bound), which is stable under the evolution of the VP system and corresponds to the standard framework for solving the Cauchy problem.

We face different kinds of difficulties: lack of compactness due to translation invariance, and possibility of dichotomy in the large-time dispersive regime due to the invariance under Galilean translations. The possible regimes are much richer in the gravitational case than in the plasma physics case (see for instance [10] for the construction of time-periodic solutions). This also makes the analysis, for instance of the dispersive regime, much harder than in the plasma physics case $[9,15,5]$. 


\section{Optimal bounds for the kinetic and potential en- ergies}

The goal of this section is to determine an optimal interval for the kinetic and potential energies in terms of the total energy, the total mass being fixed. We reduce the question to a minimization problem and prove that it is achieved.

Let $f(t, x, v)$ be a solution of the VP system and define the total energy associated to $f$ by

$$
E(f):=E_{K I N}(f)-\gamma E_{P O T}(f)
$$

where the kinetic and the potential energies are defined respectively by

$$
E_{K I N}(f)=\frac{1}{2} \int_{\mathbb{R}^{6}}|v|^{2} f(t, x, v) d x d v \quad \text { and } \quad E_{P O T}(f)=\frac{1}{8 \pi} \int_{\mathbb{R}^{3}}|\nabla \phi|^{2} d x .
$$

In the last expression the potential $\phi$ associated to $f$ is given by

$$
\phi=-\frac{\gamma}{|\cdot|} * \int_{\mathbb{R}^{3}} f(\cdot, v) d v .
$$

For a smooth solution the total energy remains constant along the time evolution of the solution as well as the total mass which is defined by

$$
\|f(t, \cdot, \cdot)\|_{\mathrm{L}^{1}\left(\mathbb{R}^{6}\right)}=\int_{\mathbb{R}^{6}} f(t, x, v) d x d v
$$

(see $[8,15])$. The transport of the distribution function also preserves uniform bounds:

$$
\|f(t, \cdot, \cdot)\|_{\mathrm{L}^{\infty}\left(\mathbb{R}^{6}\right)} \leq\|f(0, \cdot, \cdot)\|_{\mathrm{L}^{\infty}\left(\mathbb{R}^{6}\right)} .
$$

For these reasons, it is natural to consider the functional space $\mathrm{L}^{1} \cap \mathrm{L}^{\infty}\left(\mathbb{R}^{6}\right)$. Our main result relates $E(f), E_{K I N}(f)$ and $E_{P O T}(f)$ in this functional space. It is by the way independent of the VP system itself but of course applies to any of its solutions. Before, we need some further notations and definitions. For any $M>0$, let

$$
\Gamma_{M}=\left\{f \in \mathrm{L}^{1} \cap \mathrm{L}^{\infty}\left(\mathbb{R}^{6}\right): f(x, v) \geq 0,\|f\|_{\mathrm{L}^{1}\left(\mathbb{R}^{6}\right)}=M,\|f\|_{\mathrm{L}^{\infty}\left(\mathbb{R}^{6}\right)} \leq 1\right\}
$$

and consider

$$
E_{M}:=\inf \left\{E(f): f \in \Gamma_{M}\right\} .
$$

In the rest of this paper, we will assume without further notice that $\gamma=1$ (gravitational case). For any $E \geq E_{M}$, define

$$
\begin{aligned}
& K_{ \pm}(E, M)=-2 E_{M}\left(1-\frac{E}{2 E_{M}} \pm \sqrt{1-\frac{E}{E_{M}}}\right) \\
& P_{ \pm}(E, M)=-2 E_{M}\left(1 \pm \sqrt{1-\frac{E}{E_{M}}}\right)
\end{aligned}
$$

Theorem $1 E_{M}$ is negative, bounded from below and for any $f \in \Gamma_{M}$, with $E=E(f)$, the following properties hold:

(i) $E_{K I N}(f) \in\left[K_{-}(E, M), K_{+}(E, M)\right]$

(ii) $E_{P O T}(f) \in\left[\max \left\{0, P_{-}(E, M)\right\}, P_{+}(E, M)\right]$

(iii) $E_{P O T}(f) \in\left[0, \sqrt{-4 E_{M} E_{K I N}(f)}\right]$ 
Moreover, there exist functions which minimize (2) and these are stationary solutions to the VP system for which $E=E_{M}$ and

$$
K_{ \pm}(E, M)=E_{K I N}(f)=\frac{1}{2} E_{P O T}(f)=P_{ \pm}(E, M) .
$$

The rest of this section is devoted to the proof of Theorem 1, apart the fact that minimizers are solutions of the VP system, which is going to be an easy consequence of the explicit form of the minimizers (see Theorem 2 in Section 3).

\subsection{A potential energy estimate}

Lemma 1 There exists a positive constant $C$ such that for any nonnegative function $f$ in $\mathrm{L}^{1} \cap \mathrm{L}^{\infty}\left(\mathbb{R}^{6}\right)$ with $|v|^{2} f \in L^{1}\left(\mathbb{R}^{6}\right)$ and $\phi$ given by (1),

$$
\int_{\mathbb{R}^{3}}|\nabla \phi|^{2} d x \leq C\|f\|_{\mathrm{L}^{1}\left(\mathbb{R}^{6}\right)}^{7 / 6}\|f\|_{\mathrm{L}^{\infty}\left(\mathbb{R}^{6}\right)}^{1 / 3}\left(\int_{\mathbb{R}^{6}}|v|^{2} f(x, v) d x d v\right)^{1 / 2} .
$$

In the rest of this paper, we shall denote by $\mathcal{C}$ the best constant in Inequality (3) (see Appendix B for more details).

Proof. From the definition of $\phi$ we have

$$
\int_{\mathbb{R}^{3}}|\nabla \phi|^{2} d x=\int_{\mathbb{R}^{3}}(-\Delta \phi) \phi d x=4 \pi \int_{\mathbb{R}^{6}} \frac{\rho(y) \rho(x)}{|x-y|} d x d y
$$

with $\rho(x)=\int_{\mathbb{R}^{3}} f(x, v) d v$. According to the Hardy-Littlewood-Sobolev inequalities,

$$
\int_{\mathbb{R}^{3}}|\nabla \phi|^{2} d x \leq 4 \pi \Sigma\|\rho\|_{L^{\frac{6}{5}}\left(\mathbb{R}^{3}\right)}^{2}
$$

for some constant $\Sigma>0$. Because of Hölder's inequality,

$$
\|\rho\|_{L^{6 / 5}\left(\mathbb{R}^{3}\right)} \leq\|\rho\|_{\mathrm{L}^{1}\left(\mathbb{R}^{3}\right)}^{7 / 12}\|\rho\|_{\mathrm{L}^{5 / 3}\left(\mathbb{R}^{3}\right)}^{5 / 2} .
$$

The $\mathrm{L}^{5 / 3}$-norm of $\rho$ can be estimated by the standard interpolation inequality

$$
\int_{\mathbb{R}^{3}}|\rho|^{5 / 3} d x \leq C\|f\|_{L^{\infty}\left(\mathbb{R}^{6}\right)}^{2 / 3} \int_{\mathbb{R}^{6}}|v|^{2} f(x, v) d x d v .
$$

\subsection{An equivalent minimization problem}

Define

$$
J(f)=\frac{\frac{1}{2} \int_{\mathbb{R}^{6}}|v|^{2} f d x d v}{\left(\frac{1}{8 \pi} \int_{\mathbb{R}^{6}}|\nabla \phi|^{2} d x\right)^{2}} \equiv \frac{E_{K I N}(f)}{\left(E_{P O T}(f)\right)^{2}}
$$

and consider the minimization problem

$$
J_{M}=\inf \left\{J(f): f \in \Gamma_{M}\right\} .
$$

The strict positive character of $J_{M}$ is a trivial consequence of the Inequality (3). A simple scaling argument proves that the constraint $\|f\|_{\mathrm{L}^{\infty}\left(\mathbb{R}^{6}\right)} \leq 1$ has to be saturated.

Lemma 2 The minimization problems $E(f)=E_{M}$ and $J(f)=J_{M}$ over the set $\Gamma_{M}$ are equivalent in the following sense.

(i) Their respective minima satisfy

$$
4 J_{M} E_{M}=-1 .
$$


(ii) If $f_{M} \in \Gamma_{M}$ is a minimizer of the functional $E$, then it is also a minimizer of the functional $J$. On the other hand, if $J\left(g_{M}\right)=J_{M}$ for some $g_{M} \in \Gamma_{M}$, then $E\left(g_{M}^{\sigma}\right)=E_{M}$ where $g_{M}^{\sigma}(x, v):=g_{M}(\sigma x, v / \sigma)$ and $\sigma=\frac{E_{P O T}\left(g_{M}\right)}{2 E_{K I N}\left(g_{M}\right)}$.

Proof. The set $\Gamma_{M}$ is stable under the action of the scaling $f \mapsto f^{\sigma}(x, v)=f(\sigma x, v / \sigma)$ for any $\sigma>0$. Since for every $f \in \Gamma_{M}$,

$$
E\left(f^{\sigma}\right)=\sigma^{2} E_{K I N}(f)-\sigma E_{P O T}(f),
$$

we can select the value of the parameter $\sigma$ for which the total energy reaches the minimum over the uniparametric family of functions $\left\{f^{\sigma}: \sigma \in \mathbb{R}^{+}\right\}$. Let

$$
\sigma=\sigma_{\min }=\frac{E_{P O T}(f)}{2 E_{K I N}(f)} .
$$

In that case,

$$
E(f) \geq E\left(f^{\sigma_{\min }}\right)=-\frac{1}{4} \frac{\left(E_{P O T}(f)\right)^{2}}{E_{K I N}(f)}=-\frac{1}{4 J(f)} .
$$

Note that $E\left(f^{\sigma_{\text {min }}}\right)<0$. Since $-\frac{1}{4 J(f)} \geq-\frac{1}{4 J_{M}}$, this proves that $E_{M} \geq-\frac{1}{4 J_{M}}$. On the other hand, the functional $J$ is invariant under scalings. so we may rewrite (5) as

$$
J(f)=J\left(f^{\sigma_{\min }}\right)=-\frac{1}{4 E\left(f^{\sigma_{\min }}\right)} .
$$

Again $-\frac{1}{4 E\left(f^{\left.\sigma_{\text {min }}\right)}\right.} \geq-\frac{1}{4 E_{M}}$ proves the inequality: $J_{M} \geq-\frac{1}{4 E_{M}}$, so that $E_{M} \leq-\frac{1}{4 J_{M}}$ because $E_{M}<0$. Assertions concerning the minimizers directly follow from (5) and (6).

The fact that $E_{M}$ is negative, bounded from below, is a straightforward consequence of Lemma 1 and Lemma 2. We can now prove Assertions (i)-(iii) of Theorem 1. By definition of $E(f)$ and $J(f)$, we have

$$
E:=E(f)=E_{K I N}-E_{P O T} \quad \text { and } \quad \frac{E_{K I N}(f)}{\left(E_{P O T}(f)\right)^{2}}=J(f) \geq-\frac{1}{4 E_{M}} .
$$

This proves Assertion (iii): $\left(E_{P O T}(f)\right)^{2} \leq-4 E_{M} E_{K I N}(f)$, and

$$
-\frac{\left.E_{P O T}(f)\right)^{2}}{4 E_{M}}-E_{P O T}(f) \leq E, \quad\left(E_{K I N}-E\right)^{2} \leq-4 E_{M} E_{K I N},
$$

from which (i) and (ii) easily follow, using the positivity of $E_{P O T}(f)$. Note that $K_{-}(E, M)$ is nonnegative, which is the case for $P_{-}(E, M)$ only if $E<0$.

The rest of this section is devoted to the proof of the existence of minimizers.

Corollary 3 Let $f_{M}$ be a minimizing function for the functional $E$ on $\Gamma_{M}$. Then

$$
E_{P O T}\left(f_{M}\right)=2 E_{K I N}\left(f_{M}\right)=-2 E_{M} .
$$

Proof. (7) is a trivial consequence of the scaling argument (4): derive the identity with respect to $\sigma$ at $\sigma=1$. Note that

$$
E_{M}=-E_{K I N}\left(f_{M}\right)=-\frac{1}{2} E_{P O T}\left(f_{M}\right)<0 .
$$

Remark Property (7) is shared by any stationary solution $f$ of the VP system:

$$
E_{P O T}(f)=2 E_{K I N}(f)=-2 E(f) .
$$

For a proof, see identity (23). 


\subsection{Spherical symmetry and regularity of the potential}

We first prove by symmetric nonincreasing rearrangements (see Appendix A) that when minimizing the functional $E$ on $\Gamma_{M}$, we can consider minimizing sequences having radial nonincreasing mass densities, which provides further regularity properties of the associated potentials.

Lemma 4 Let $M>0$. There exists a minimizing sequence $\left(f_{n}\right)_{n \in \mathbb{N}} \in \Gamma_{M}^{\mathbb{N}}$ of the functional $E$ such that for any $n \in \mathbb{N}$ the mass density $\rho_{n}(x)=\int_{\mathbb{R}^{3}} f_{n}(x, v) d v$ is a radial nonincreasing function.

Proof. Let $\left(f_{n}\right)_{n \in \mathbb{N}} \in \Gamma_{M}^{\mathbb{N}}$ be an arbitrary minimizing sequence of the functional $E$. The symmetric nonincreasing rearrangement $f_{n}^{*_{x}} \in \Gamma_{M}$ (see Appendix A) of $f_{n}$ (with respect to the $x$ variable only) also belongs to $\Gamma_{M}$ for any $n \in \mathbb{N}$, because of (27)-(28). Using Riesz' theorem (see Theorem 4 in Appendix A for a statement) we get

$$
\begin{aligned}
\int_{\mathbb{R}^{3}}|\nabla \phi|^{2} d x & =4 \pi \int_{\left(\mathbb{R}^{3}\right)^{4}} \frac{f(x, v) f\left(x^{\prime}, v^{\prime}\right)}{\left|x-x^{\prime}\right|} d x d x^{\prime} d v d v^{\prime} \\
& \leq 4 \pi \int_{\left(\mathbb{R}^{3}\right)^{4}} \frac{f^{*_{x}}(x, v) f^{*_{x}}\left(x^{\prime}, v^{\prime}\right)}{\left|x-x^{\prime}\right|} d x d x^{\prime} d v d v^{\prime} \\
& \leq \int_{\mathbb{R}^{3}}\left|\nabla \phi_{*}\right|^{2} d x
\end{aligned}
$$

where $\phi=|\cdot|^{-1} * \int_{\mathbb{R}^{3}} f(\cdot, v) d v$ and $\phi_{*}=|\cdot|^{-1} * \int_{\mathbb{R}^{3}} f^{* x}(\cdot, v) d v$. This and (29) prove that $f_{n}^{*_{x}}$ is a minimizing sequence. Properties (30)-(31) provide the spherically symmetric and nonincreasing character of the sequence of mass densities associated to $f_{n}^{*_{x}}$.

The spherically symmetric character of the mass density implies regularity properties of the potential function $\phi$ (see Lemma 2 of [6] for a proof) which go beyond the estimate of Lemma 1.

Lemma 5 Let $\rho \in \mathrm{L}^{1}\left(\mathbb{R}^{3}\right) \cap \mathrm{L}^{5 / 3}\left(\mathbb{R}^{3}\right)$ be a nonnegative and spherically symmetric function with $\|\rho\|_{\mathrm{L}^{1}\left(\mathbb{R}^{3}\right)}=M>0$ and define $\phi=-|\cdot|^{-1} * \rho$. Then $\phi$ belongs to $W_{\mathrm{loc}}^{2,5 / 3}\left(\mathbb{R}^{3}\right)$ and there exists a $\eta>0$ such that for any $R>0$ we have

$$
\int_{|x|<R}|\nabla \phi|^{2+\eta} d x \leq C(M, R)\left(\int_{|x|<R} \rho^{5 / 3} d x+1\right)
$$

for some $C=C(M, R)>0$ which does not depend on $\rho$.

\subsection{A priori estimates, scalings and tools of the concentration- compactness method}

Several of the results of this paragraph are basic tools of the concentration-compactness method (see $[14,16]$ for more details in this direction). We start with a very elementary computation which will be usefull later.

Lemma 6 Let $\rho$ be a radial $L^{1}$ nonnegative nontrivial function on $\mathbb{R}^{3}$ and consider the corresponding potential $\phi$ given by the Poisson equation

$$
\Delta \phi=4 \pi \rho, \quad \lim _{|x| \rightarrow+\infty} \phi(x)=0 .
$$

Then $\phi$ is radial, nondecreasing and strictly increasing in the interior of the support of $\rho$. With the notation $\int_{\mathbb{R}^{3}} \rho(x) d x=: M>0$ and the standard abuse of notations: $r=|x|, \rho(x)=\rho(r), \phi(x)=\phi(r)$ for any $x \in \mathbb{R}^{3}$ and $M=\int_{\mathbb{R}^{3}} \rho(x) d x=4 \pi \int_{0}^{\infty} r^{2} \rho(r)$, the two following estimates hold: 
(i) For any $r>0$,

$$
\phi^{\prime}(r) \leq \frac{M}{r^{2}} \quad \text { and } \quad-\frac{M}{r} \leq \phi(r) \leq 0
$$

(ii) For any $R>0$,

$$
\int_{|x| \geq R}|\nabla \phi(x)|^{2} d x \leq 4 \pi \frac{M^{2}}{R} .
$$

Proof. The Poisson equation written in radial coordinates is

$$
\frac{1}{r^{2}}\left(r^{2} \phi^{\prime}\right)^{\prime}=4 \pi \rho
$$

which gives after one integration

$$
\phi^{\prime}(r)=\frac{4 \pi}{r^{2}} \int_{0}^{r} s^{2} \rho(s) d s \leq \frac{M}{r^{2}} .
$$

An integration from $R>0$ to $+\infty$ gives (i) while (ii) is obtained by writing

$$
\int_{|x| \geq R}|\nabla \phi(x)|^{2} d x \leq 4 \pi \int_{R}^{+\infty} r^{2}\left(\phi^{\prime}(r)\right)^{2} d r \leq 4 \pi \int_{R}^{+\infty} \frac{M^{2}}{r^{2}} d r .
$$

The bound on $\phi$ readily follows from the expression of $\phi^{\prime}$.

Our next result is based on a scaling argument.

Lemma 7 Let $M$ be a positive real number. Then, the identity

$$
E_{M}=M^{7 / 3} E_{1}
$$

holds.

Proof. Let $f \in \Gamma_{1}$. We scale this function as $\bar{f}(x, v)=f\left(M^{1 / 3} x, M^{-2 / 3} v\right)$, obtaining

$$
\|\bar{f}\|_{\mathrm{L}^{1}\left(\mathbb{R}^{6}\right)}=M, \quad\|\bar{f}\|_{\mathrm{L}^{\infty}\left(\mathbb{R}^{6}\right)} \leq 1, \quad E(\bar{f})=M^{7 / 3} E(f) .
$$

This scaling trivially implies (8).

The following result is a splitting estimate (see [6] for similar estimates).

Lemma 8 Let $f \in \Gamma_{M}$ be a function such that the mass density $\rho(x)=\int_{\mathbb{R}^{3}} f(x, v) d v$ is spherically symmetric. Given $R>0$, we can write

$$
M-\lambda=\int_{|x|<R} \int_{\mathbb{R}^{3}} f(x, v) d v d x
$$

for some $\lambda \in[0, M]$. Then

$$
E(f)-E_{M} \geq-\left(\frac{7}{3} \frac{E_{M}}{M^{2}}+\frac{1}{4 \pi R}\right)(M-\lambda) \lambda .
$$

Proof. Let $\chi_{B_{R}}$ be the characteristic function of $B_{R}:=\left\{x \in \mathbb{R}^{3}:|x|<R\right\}$. We split the potential function in two parts $\phi=\phi_{1}+\phi_{2}$, where $\phi_{1}$ and $\phi_{2}$ are defined by

$$
\Delta \phi_{1}(x)=\int_{\mathbb{R}^{3}} \chi_{B_{R}}(x) f(x, v) d v, \quad \Delta \phi_{2}(x)=\int_{\mathbb{R}^{3}}\left(1-\chi_{B_{R}}(x)\right) f(x, v) d v .
$$


In the same line we write $E(f)$ as

$$
\begin{aligned}
E(f)= & E_{K I N}\left(\chi_{B_{R}} f\right)+E_{K I N}\left(\left(1-\chi_{B_{R}}\right) f\right) \\
& -\frac{1}{8 \pi} \int_{\mathbb{R}^{3}}\left|\nabla \phi_{1}\right|^{2} d x-\frac{1}{8 \pi} \int_{\mathbb{R}^{3}}\left|\nabla \phi_{2}\right|^{2} d x-\frac{1}{4 \pi} \int_{\mathbb{R}^{3}} \nabla \phi_{1} \cdot \nabla \phi_{2} d x \\
= & E\left(\chi_{B_{R}} f\right)+E\left(\left(1-\chi_{B_{R}}\right) f\right)-\frac{1}{4 \pi} \int_{\mathbb{R}^{3}} \nabla \phi_{1} \cdot \nabla \phi_{2} d x \\
\geq & E_{M-\lambda}+E_{\lambda}+\frac{1}{4 \pi} \int_{\mathbb{R}^{3}} \phi_{2} \Delta \phi_{1} d x .
\end{aligned}
$$

Using (8) we find

$$
E(f)-E_{M} \geq\left[\left(1-\frac{\lambda}{M}\right)^{\frac{7}{3}}+\left(\frac{\lambda}{M}\right)^{\frac{7}{3}}-1\right] E_{M}+\frac{1}{4 \pi} \int_{\mathbb{R}^{3}} \phi_{2} \Delta \phi_{1} d x .
$$

In order to bound the first term on the right hand side of (10) we take advantage of the negative value of $E_{M}$ and use the identity

$$
(1-x)^{7 / 3}+x^{7 / 3}-1 \leq-\frac{7}{3} x(1-x),
$$

which is valid for all $x$ in $[0,1]$. The second term of the right hand side of $(10)$ is nonpositive and bounded by

$$
\left|\frac{1}{4 \pi} \int_{\mathbb{R}^{3}} \phi_{2} \Delta \phi_{1} d x\right| \leq\left\|\phi_{2}\right\|_{L^{\infty}\left(\mathbb{R}^{3}\right)} \int_{\mathbb{R}^{6}} \chi_{B_{R}} f d x d v=\left\|\phi_{2}\right\|_{L^{\infty}\left(\mathbb{R}^{3}\right)}(M-\lambda),
$$

where $\left\|\phi_{2}\right\|_{\mathrm{L}^{\infty}\left(\mathbb{R}^{3}\right)}$ can be calculated by using the spherically symmetric character of the mass density $\rho=\int_{\mathbb{R}^{3}} f d v$ :

$$
\phi_{2}^{\prime}(r)=\frac{4 \pi}{r^{2}} \int_{0}^{r} s^{2} \rho(s)\left(1-\chi_{B_{R}}(s)\right) d s \geq 0,
$$

so that $\phi_{2}^{\prime}(r) \equiv 0$ on $(0, R)$, which implies that

$$
\left\|\phi_{2}\right\|_{L^{\infty}\left(\mathbb{R}^{3}\right)}=\left|\phi_{2}(0)\right|=\left|\phi_{2}(R)\right| \leq \frac{\lambda}{4 \pi R}
$$

according to Lemma 6 . Combining the above estimates we obtain (9).

In the next lemma we prove that no vanishing of mass occurs.

Lemma 9 Let $R_{0}>\frac{3 M^{2}}{28 \pi\left|E_{M}\right|}$ and consider a minimizing sequence $\left(f_{n}\right)_{n \in \mathbb{N}} \in \Gamma_{M}^{\mathbb{N}}$ for the functional $E$. Assume moreover that $\left(f_{n}\right)_{n \in \mathbb{N}}$ is given as in Lemma 4. Then

$$
\limsup _{n \rightarrow \infty} \int_{|x| \geq R_{0}} \int_{\mathbb{R}^{3}} f_{n} d v d x=0 .
$$

Proof. If the statement was not true, there would exist a $\lambda \in(0, M]$ and a subsequence (we keep the same notation for the sake of simplicity) such that

$$
\lim _{n \rightarrow \infty} \int_{|x| \geq R_{0}} \int_{\mathbb{R}^{3}} f_{n} d v d x=\lambda
$$

In this case, for every $f_{n}$, there would exist $R(n)>R_{0}$ such that

$$
\frac{\lambda}{2}=\int_{|x| \geq R(n)} \int_{\mathbb{R}^{3}} f_{n} d v d x .
$$


Apply now Lemma 8 to each $f_{n}$ with $R=R(n)$ :

$$
\begin{aligned}
E\left(f_{n}\right)-E_{M} & \geq-\left(\frac{7 E_{M}}{3 M^{2}}+\frac{1}{4 \pi R(n)}\right)\left(M-\frac{\lambda}{2}\right) \frac{\lambda}{2} \\
& \geq-\left(\frac{7 E_{M}}{3 M^{2}}+\frac{1}{4 \pi R_{0}}\right)\left(M-\frac{\lambda}{2}\right) \frac{\lambda}{2}>0 .
\end{aligned}
$$

This would clearly be in contradiction with the assumption that the sequence is a minimizing sequence for the functional $E$.

\subsection{Convergence of a minimizing sequence}

Proposition 10 Let $\left(f_{n}\right)_{n \in \mathbb{N}} \in \Gamma_{M}^{\mathbb{N}}$ be a minimizing sequence for the functional $E$, with radial nonincreasing mass densities. Up to a subsequence, the sequence converges to a minimizer $f_{M} \in \Gamma_{M}$ such that $E_{M}=E\left(f_{M}\right), \operatorname{supp}\left(f_{M}\right) \subset B_{R_{0}} \times \mathbb{R}^{3}$ where $R_{0}=\frac{3 M^{2}}{28 \pi\left|E_{M}\right|}$.

Proof. At each step of the proof, we may extract subsequences that we still index by $n$, for simplicity. From Lemma 1 , it is clear that both $E_{K I N}\left(f_{n}\right)$ and $E_{P O T}\left(f_{n}\right)$ are bounded sequences. Thanks to Lemma $9, \lim _{n \rightarrow \infty} \int_{B_{R_{0}}} \int_{\mathbb{R}^{3}} f_{n} d v d x=M$. The sequence $\left(f_{n}\right)_{n \in \mathbb{N}}$ verifies the hypothesis of the Dunford-Pettis theorem:

(i) [boundedness] $\left(f_{n}\right)_{n \in \mathbb{N}}$ is bounded in $\mathrm{L}^{1}\left(\mathbb{R}^{6}\right)$,

(ii) [no concentration] for any measurable set $A$,

$$
\int_{A} f_{n} d x d v \leq\left\|f_{n}\right\|_{\mathrm{L}^{\infty}\left(\mathbb{R}^{6}\right)}|A| \leq|A|,
$$

(iii) [no vanishing] for any $K_{1}, K_{2}$, either $K_{1}<R_{0}$ and

$$
\int_{|x|>K_{1}} \int_{|v|>K_{2}} f_{n} d x d v \leq \int_{\mathbb{R}^{3}} \int_{|v|>K_{2}} f_{n} d x d v \leq \frac{1}{K_{2}^{2}} E_{K I N}\left(f_{n}\right),
$$

or $K_{1} \geq R_{0}$ and

$$
\lim _{n \rightarrow \infty} \int_{|x|>K_{1}} \int_{|v|>K_{2}} f_{n} d x d v \leq \lim _{n \rightarrow \infty} \int_{|x| \geq R_{0}} \int_{\mathbb{R}^{3}} f_{n} d x d v=0 .
$$

As a consequence, there exists a function $f \in \mathrm{L}^{1}\left(\mathbb{R}^{6}\right)$ and a subsequence which weakly converges in $\mathrm{L}^{1}\left(\mathbb{R}^{6}\right)$ to $f$. As a consequence, $\|f\|_{\mathrm{L}^{1}\left(\mathbb{R}^{6}\right)}=M$ (see $[14,6]$ for more details). Moreover, $f$ is nonnegative a.e. as a weak limit of nonnegative functions. The sequence $\left(f_{n}\right)_{n \in \mathbb{N}}$ is bounded in $\mathrm{L}^{\infty}\left(\mathbb{R}^{3}\right)$ and thus also converges to $f$ w.r.t. the ${ }^{*}$-weak $\mathrm{L}^{\infty}$ topology, up to the extraction of a further subsequence, so that $\|f\|_{\mathrm{L}^{\infty}\left(\mathbb{R}^{3}\right)} \leq 1$. Thus $f$ belongs to $\Gamma_{M}$. The weak convergence in $\mathrm{L}^{1}\left(\mathbb{R}^{6}\right)$ implies

$$
\int_{\mathbb{R}^{6}}|v|^{2} f d x d v \leq \liminf _{n \rightarrow \infty} \int_{\mathbb{R}^{6}}|v|^{2} f_{n} d x d v .
$$

Let $\phi_{n}$ and $\phi$ be the solutions to the Poisson equation with mass densities associated with $f_{n}$ and $f$ respectively. The proof that $\lim _{n \rightarrow \infty}\left\|\nabla \phi_{n}-\nabla \phi\right\|_{\mathrm{L}^{2}\left(\mathbb{R}^{3}\right)}=0$ up to the extraction of a subsequence follows from the splitting

$$
\int_{\mathbb{R}^{3}}\left|\nabla \phi_{n}-\nabla \phi\right|^{2} d x \leq \int_{B_{R}}\left|\nabla \phi_{n}-\nabla \phi\right|^{2} d x+4 \pi \frac{M^{2}}{R},
$$

which is itself a consequence of Lemma 6 . Here $B_{R}:=\left\{x \in \mathbb{R}^{3}:|x|<R\right\}$. From Lemma 5 and the Sobolev compact inclusion $W^{2,5 / 3}\left(B_{R}\right) \hookrightarrow W^{1, \frac{15}{4}}\left(B_{R}\right)$, we obtain the convergence by choosing $R$ large enough in (11). This proves that $E(f)=E_{M}$. 


\section{Solutions of the VP system with minimal energy and nonlinear stability}

We characterize the functions with minimal energy and prove that they are solutions of the VP system. For that purpose, we characterize the mass density of a minimizer, as was proposed in [16].

Theorem 2 Let $f_{M}$ be a minimizing function for the functional $E$ on $\Gamma_{M}$, with radial mass density. Then $f_{M}$ is defined almost everywhere by

$$
f_{M}(x, v)=\left\{\begin{array}{l}
1 \quad \text { if } \frac{1}{2}|v|^{2}+\phi_{f_{M}}(x)<\frac{7}{3} \frac{E\left(f_{M}\right)}{M}, \\
0 \quad \text { otherwise }
\end{array}\right.
$$

where $\phi_{f_{M}}$ is the unique radial solution on $\mathbb{R}^{3}$ of

$$
\Delta \phi_{f_{M}}=\frac{1}{3}(4 \pi)^{2}\left[2\left(\frac{7}{3} \frac{E_{M}}{M}-\phi_{f_{M}}\right)_{+}\right]^{3 / 2} .
$$

It is the unique minimizer with radial mass density and it is also a steady-state solution to the VP system. Moreover, if $f$ is another minimizing function, then

$$
f(x, v)=f_{M}(x-\bar{x}, v) \quad \forall(x, v) \in \mathbb{R}^{6},
$$

where $\bar{x}=\frac{1}{M} \int_{\mathbb{R}^{6}} x f(x, v) d x d v$.

Here $w_{+}$denotes the positive part of $w$. The existence of a minimum implies by translation in space the existence of other ones. The fact $f_{M}$ is a solution to the VP system is a straightforward consequence of the fact that $f_{M}$ is a function of the microscopic energy: $\frac{1}{2}|v|^{2}+\phi_{f_{M}}(x)$, namely

$$
f_{M}(x, v)=\chi_{\frac{1}{2}|v|^{2}+\phi_{f_{M}}(x)<\frac{7}{3} \frac{E\left(f_{M}\right)}{M}}(x, v) .
$$

In this section, we first prove Theorem 2 and then state a nonlinear stability result for the solutions of the VP system.

\subsection{Explicit form of the minimizers}

For convenience, we split the proof of Theorem 2 into three intermediate results.

Lemma 11 Let $f_{M}$ be a minimizing function for the functional $E$ on $\Gamma_{M}$. Then

$$
f_{M}(x, v)= \begin{cases}1 & \text { for }(x, v) \text { such that }|v| \leq\left(\frac{3}{4 \pi} \rho_{M}(x)\right)^{1 / 3} \text { a.e. } \\ 0 & \text { otherwise }\end{cases}
$$

Note that we do not assume that $f_{M}$ has a radial mass density.

Proof. We are going to split the proof of (12) into several steeps. First, we observe that

$$
\left\|f_{M}\right\|_{\mathrm{L}^{\infty}\left(\mathbb{R}^{6}\right)}=1 .
$$

If this is not the case, consider the scaling $\bar{f}(x, v)=\kappa f\left(\kappa^{2 / 3} x, \kappa^{-1 / 3} v\right)$, which gives

$$
\|\bar{f}\|_{L^{1}\left(\mathbb{R}^{6}\right)}=\|f\|_{L^{1}\left(\mathbb{R}^{6}\right)}, \quad\|\bar{f}\|_{L^{\infty}\left(\mathbb{R}^{6}\right)}=\kappa\|f\|_{L^{\infty}\left(\mathbb{R}^{6}\right)}, \quad E(\bar{f})=\kappa^{2 / 3} E(f) .
$$

By applying this scaling to $f=f_{M}$ with $\kappa=\left\|f_{M}\right\|_{\mathrm{L}^{\infty}\left(\mathbb{R}^{6}\right)}^{-1}>1$, we would get $E(\bar{f})=$ $\kappa^{\frac{2}{3}} E_{M}<E_{M}$ (remind that $E_{M}<0$ ), a contradiction. 
Using the Euler-Lagrange multipliers method, we are now going to prove that

$$
f_{M} \equiv 1 \text { a.e. on } \operatorname{supp}\left(f_{M}\right) \text {. }
$$

Let $\epsilon \in(0,1)$ be a fixed real number. Let $g(x, v) \in \mathrm{L}^{1}\left(\mathbb{R}^{6}\right) \cap \mathrm{L}^{\infty}\left(\mathbb{R}^{6}\right)$ be a test function such that $g \geq 0$ a.e. in $\mathbb{R}^{6} \backslash \operatorname{supp}\left(f_{M}\right)$, with compact support contained inside

$$
\left(\operatorname{supp}\left(f_{M}\right) \backslash S_{\epsilon}\right)^{c} \equiv\left(\mathbb{R}^{6} \backslash \operatorname{supp}\left(f_{M}\right)\right) \cup S_{\epsilon},
$$

where

$$
S_{\epsilon}=\left\{(x, v) \in \mathbb{R}^{6}: \epsilon \leq f_{M}(x, v) \leq 1-\epsilon\right\} .
$$

With $T:=M \epsilon\left(M\|g\|_{\mathrm{L}^{\infty}\left(\mathbb{R}^{6}\right)}+\|g\|_{\mathrm{L}^{1}\left(\mathbb{R}^{6}\right)}\right)^{-1}$, we have that

$$
g(t)=M \frac{t g+f_{M}}{\left\|t g+f_{M}\right\|_{\mathrm{L}^{1}\left(\mathbb{R}^{6}\right)}} \in \Gamma_{M} \quad \forall t \in[0, T] .
$$

The function $g$ depends on $t, x$ and $v$. However, to emphasize the dependence in $t$, we will write it $g(t)$. Identity (13) follows from a detailed analysis of the function $t g+f_{M}$ :

$$
\begin{aligned}
& 0 \leq-T\|g\|_{\mathrm{L}^{\infty}\left(\mathbb{R}^{6}\right)}+\epsilon \leq t g+f_{M} \quad \text { in } S_{\epsilon}, \\
& 0 \leq \quad f_{M} \quad=t g+f_{M} \quad \text { in } \operatorname{supp}\left(f_{M}\right) \backslash S_{\epsilon} \text {, } \\
& 0 \leq \quad t g \quad=t g+f_{M} \quad \text { in } \mathbb{R}^{6} \backslash \operatorname{supp}\left(f_{M}\right) \text {, }
\end{aligned}
$$

gives the positivity of $g(t)$ and implies

$$
M(1-\epsilon) \leq\left\|t g+f_{M}\right\|_{\mathrm{L}^{1}\left(\mathbb{R}^{6}\right)}=t \int_{\mathbb{R}^{6}} g d x d v+M \leq M(1+\epsilon) .
$$

It is clear that $\|g(t)\|_{\mathrm{L}^{1}\left(\mathbb{R}^{6}\right)}=M$ and the estimate

$$
\|g(t)\|_{L^{\infty}\left(S_{\epsilon}\right)} \leq M \frac{T\|g\|_{L^{\infty}\left(\mathbb{R}^{6}\right)}+1-\epsilon}{M-T\|g\|_{L^{1}\left(\mathbb{R}^{6}\right)}}=1
$$

ends the proof of (13).

To prove that $S_{\epsilon}$ is a set of measure 0 for any $\epsilon>0$, we compute $E(g(t))-E_{M}=$ $E(g(t))-E\left(f_{M}\right)$ and then derive it with respect to $t$ at $t=0_{+}$. Deriving $g(t)$ with respect to $t$, we get

$$
\begin{aligned}
\frac{g^{\prime}(t)}{M} & =\frac{g}{\left\|t g+f_{M}\right\|_{\mathrm{L}^{1}\left(\mathbb{R}^{6}\right)}}-\frac{\left(t g+f_{M}\right) \int_{\mathbb{R}^{6}} g d x d v}{\left\|t g+f_{M}\right\|_{\mathrm{L}^{1}\left(\mathbb{R}^{6}\right)}^{2}} \\
\frac{g^{\prime \prime}(t)}{M} & =-2 \frac{g \int_{\mathbb{R}^{6}} g d x d v}{\left\|t g+f_{M}\right\|_{\mathrm{L}^{1}\left(\mathbb{R}^{6}\right)}^{2}}+2 \frac{\left(t g+f_{M}\right)\left[\int_{\mathbb{R}^{6}} g d x d v\right]^{2}}{\left\|t g+f_{M}\right\|_{\mathrm{L}^{1}\left(\mathbb{R}^{6}\right)}^{3}} .
\end{aligned}
$$

By a Taylor expansion at $t=0_{+}$, there exists a $\theta \in(0, t)$ such that

$$
g(t)-f_{M}=t g^{\prime}(0)+\frac{t^{2}}{2} g^{\prime \prime}(\theta)=t\left(g-\frac{1}{M}\left[\int_{\mathbb{R}^{6}} g d x d v\right] f_{M}\right)+\frac{t^{2}}{2} g^{\prime \prime}(\theta),
$$

where

$$
\left|g^{\prime \prime}(\theta)\right| \leq C\left(\left|f_{M}\right|+|g|\right)
$$

for some constant $C>0$ which depends only on $f_{M}$ and $g$. Using the decomposition $E(g(t))-E_{M}=\frac{1}{2} \mathcal{K}(t)-\frac{1}{8 \pi} \mathcal{P}(t)$ with

$$
\begin{aligned}
\mathcal{K}(t) & =\int_{\mathbb{R}^{6}}|v|^{2}\left[g(t)-f_{M}\right] d x d v \\
\mathcal{P}(t) & =8 \pi \int_{\mathbb{R}^{6}} \phi_{f_{M}}\left[g(t)-f_{M}\right] d x d v-\int_{\mathbb{R}^{3}}\left[\left|\nabla \phi_{g(t)}-\nabla \phi_{f_{M}}\right|^{2}\right] d x
\end{aligned}
$$


we have therefore the following estimates:

$$
\begin{aligned}
& \left.\left.\left|\int_{\mathbb{R}^{6}}\right| v\right|^{2}\left[g(t)-f_{M}-t g^{\prime}(0)\right] d x d v\left|\leq C t^{2} \int_{\mathbb{R}^{6}}\right| v\right|^{2}\left[|g|+f_{M}\right] d x d v=O\left(t^{2}\right), \\
& \left|\int_{\mathbb{R}^{6}} \phi_{f_{M}}\left[g(t)-f_{M}-t g^{\prime}(0)\right] d x d v\right| \leq C t^{2} \int_{\mathbb{R}^{6}} \phi_{f_{M}}\left[|g|+f_{M}\right] d x d v=O\left(t^{2}\right), \\
& \int_{\mathbb{R}^{3}}\left|\nabla \phi_{g(t)}-\nabla \phi_{f_{M}}\right|^{2} d x=\int_{\mathbb{R}^{3}}\left|\nabla \phi_{g(t)-f_{M}}\right|^{2} d x=t^{2}\left\|\nabla \phi_{g^{\prime}(0)}\right\|_{\mathrm{L}^{2}\left(\mathbb{R}^{3}\right)}^{2}+O\left(t^{2}\right)=O\left(t^{2}\right) .
\end{aligned}
$$

From (7) and the above estimates we deduce

$$
\begin{aligned}
E(g(t))-E_{M} & =t \int_{\mathbb{R}^{6}}\left(\frac{1}{2}|v|^{2}+\phi_{f_{M}}\right) g^{\prime}(0) d x d v+O\left(t^{2}\right) \\
& =t \int_{\mathbb{R}^{6}}\left(\frac{1}{2}|v|^{2}+\phi_{f_{M}}\right)\left(g-\left[\frac{\int_{\mathbb{R}^{6}} g d x d v}{M}\right] f_{M}\right) d x d v+O\left(t^{2}\right) \\
& =t \int_{\mathbb{R}^{6}}\left(\frac{1}{2}|v|^{2}+\phi_{f_{M}}-\frac{3 E_{M}}{M}\right) g d x d v+O\left(t^{2}\right) .
\end{aligned}
$$

Since $f_{M}$ minimizes $E(\cdot)-E_{M}$ on $\Gamma_{M}$, we have that $E(g(t))-E_{M} \geq 0$ for any $t \in[0, T]$ and consequently

$$
\int_{\mathbb{R}^{6}}\left(\frac{1}{2}|v|^{2}+\phi_{f_{M}}-\frac{3 E_{M}}{M}\right) g d x d v \geq 0
$$

for every $g$ and $\epsilon$. There are two relevant consequences of this inequality:

(i) From the nonnegative character of $g$ on $\mathbb{R}^{6} \backslash \operatorname{supp}\left(f_{M}\right)$ we have

$$
\frac{1}{2}|v|^{2}+\phi_{f_{M}}(x) \geq \frac{3 E_{M}}{M} \quad \forall(x, v) \in \mathbb{R}^{6} \backslash \operatorname{supp}\left(f_{M}\right),
$$

or equivalently

$$
\left\{(x, v) \in \mathbb{R}^{6}: \frac{1}{2}|v|^{2}+\phi_{f_{M}}(x) \leq \frac{3 E_{M}}{M}\right\} \subset \operatorname{supp}\left(f_{M}\right) .
$$

(ii) On the other hand, $g$ has no determined sign on $S_{\epsilon}$. This implies that

$$
\frac{1}{2}|v|^{2}+\phi_{f_{M}}(x)=\frac{3 E_{M}}{M} \quad \forall(x, v) \in S_{\epsilon} \cap \operatorname{supp}\left(f_{M}\right) .
$$

The Lebesgue measure of the set defined by the above identity is zero. The set $S_{\epsilon}$ also has zero Lebesgue measure for any $\epsilon \in(0,1)$.

We conclude that $f_{M} \equiv 1$ on $\operatorname{supp}\left(f_{M}\right)$.

It remains to check that (12) holds. Since $f_{M}$ minimizes the total energy functional, it also minimizes $\left\{E(f): f \in \gamma_{M}\right\}$ where

$$
\gamma_{M}=\left\{f \in \Gamma_{M}: f \equiv 1 \text { a.e. on } \operatorname{supp}(f), \int_{\mathbb{R}^{3}} f(x, v) d v=\rho_{M}(x) \quad \forall x \in \mathbb{R}^{3}\right\} .
$$

Since all $f \in \gamma_{M}$ have the same potential energy, $E_{P O T}(f)=E_{P O T}\left(f_{M}\right)$. The problem is therefore reduced to the minimization of $\left\{E_{K I N}(f): f \in \gamma_{M}\right\}$. Using radial nonincreasing rearrangements with respect to $v$, for fixed $x \in \mathbb{R}^{3}$ (use (32) but exchange the roles of $x$ and $v$ ), we get

$$
\int_{\mathbb{R}^{3}}|v|^{2} f^{* v} d v \leq \int_{\mathbb{R}^{3}}|v|^{2} f d v
$$


with a strict inequality unless $f \equiv f^{* v}$ a.e. Thus

$$
f_{M} \equiv \chi_{|v| \leq\left(\frac{3}{4 \pi} \rho_{M}(x)\right)^{1 / 3}} \text { in } \mathbb{R}^{3} \times \mathbb{R}^{3} \text { a.e. }
$$

since, at least in the distributions sense,

$$
\rho_{M}(x)=\int_{\mathbb{R}^{3}} f_{M}(x, v) d v=\int_{\mathbb{R}^{3}} f_{M}^{* v}(x, v) d v=\frac{4 \pi}{3}\left|\operatorname{supp}\left(f_{M}\right)(x, \cdot)\right|^{3} .
$$

This conclude the proof of (12).

We have now to use the fact that $f_{M}$ is a minimizer to understand the properties of $\rho_{M}$.

Lemma 12 Let $f_{M}$ be a minimizing function for the functional $E$ on $\Gamma_{M}$ with radial mass density. Then $\rho_{M}=\int_{\mathbb{R}^{3}} f_{M}(\cdot, v) d v$ and $\phi_{f_{M}}=|\cdot|^{-1} * \rho_{M}$ are related by

$$
\rho_{M}(x)= \begin{cases}\frac{4 \pi}{3}\left[2\left(\frac{7}{3} \frac{E_{M}}{M}-\phi_{f_{M}}(x)\right)\right]^{3 / 2} & \text { if } \phi_{f_{M}}(x) \leq \frac{7}{3} \frac{E_{M}}{M} \\ 0 & \text { otherwise. }\end{cases}
$$

Proof. Let $\rho(x) \in \mathrm{L}^{1}\left(\mathbb{R}^{3}\right) \cap \mathrm{L}^{5 / 3}\left(\mathbb{R}^{3}\right)$ be a nonnegative function such that $\|\rho\|_{\mathrm{L}^{1}\left(\mathbb{R}^{3}\right)}=M$. We define

$$
f_{\rho}(x, v)= \begin{cases}1 & \text { for }(x, v) \in \mathbb{R}^{6} \text { such that }|v| \leq\left(\frac{3}{4 \pi} \rho(x)\right)^{1 / 3} \text { a.e. } \\ 0 & \text { in other case }\end{cases}
$$

We observe that $f_{\rho} \in \Gamma_{M}$. Since $f_{M}$ minimizes $E$ on $\Gamma_{M}$ and verifies (3) and (12), it also minimizes the problem

$$
\min \left\{E\left(f_{\rho}\right): \rho \in \tilde{\Gamma}_{M}\right\}
$$

where

$$
\tilde{\Gamma}_{M}:=\left\{\rho \in \mathrm{L}^{1}\left(\mathbb{R}^{3}\right) \cap \mathrm{L}^{5 / 3}\left(\mathbb{R}^{3}\right): \rho(x) \geq 0,\|\rho\|_{\mathrm{L}^{1}\left(\mathbb{R}^{3}\right)}=M\right\} .
$$

Easy computations provide

$$
\begin{aligned}
& E_{P O T}\left(f_{\rho}\right)=\frac{1}{2} \int_{\mathbb{R}^{6}} \frac{\rho(y) \rho(x)}{|x-y|} d x d y \\
& E_{K I N}\left(f_{\rho}\right)=\frac{1}{2} \int_{\mathbb{R}^{3}} \int_{\mathbb{R}^{3}}|v|^{2} f_{\rho}(x, v) d v d x=\frac{3^{5 / 3}}{10(4 \pi)^{2 / 3}} \int_{\mathbb{R}^{3}}[\rho(x)]^{5 / 3} d x,
\end{aligned}
$$

which implies that (15) can be rewritten as

$$
\min \left\{F(\rho): \rho(x) \in \tilde{\Gamma}_{M}\right\}
$$

where

$$
F(\rho):=\frac{3^{5 / 3}}{10(4 \pi)^{2 / 3}} \int_{\mathbb{R}^{3}}[\rho(x)]^{5 / 3} d x-\frac{1}{2} \int_{\mathbb{R}^{6}} \frac{\rho(y) \rho(x)}{|x-y|} d x d y .
$$

The density $\rho_{M}$ is a minimizer of (16) and therefore obeys to the corresponding EulerLagrange equations:

$$
\frac{1}{2}\left[\frac{3}{4 \pi} \rho_{M}(x)\right]^{2 / 3}-\int_{\mathbb{R} 3} \frac{\rho_{M}(y)}{|x-y|} d y-\chi=\mu
$$

where $\mu$ is a real-valued Lagrange multiplier associated to the constraint $\|\rho\|_{\mathrm{L}^{1}\left(\mathbb{R}^{3}\right)}=M$, and

$$
\begin{cases}\chi=0 & \text { if } \rho_{M}(x)>0 \\ \chi \geq 0 & \text { if } \rho_{M}(x)=0\end{cases}
$$


Multiplying (17) by $\rho_{M}$, integrating and using (7), we can obtain the value of $\mu$ :

$$
\begin{aligned}
\mu M & =\frac{1}{2}\left(\frac{3}{4 \pi}\right)^{2 / 3} \int_{\mathbb{R}^{3}}\left[\rho_{M}(x)\right]^{5 / 3} d x-\int_{\mathbb{R} 6} \frac{\rho_{M}(y) \rho_{M}(x)}{|x-y|} d x d y \\
& =\frac{5}{3} E_{K I N}\left(f_{M}\right)-2 E_{P O T}\left(f_{M}\right)=\frac{7}{3} E_{M}
\end{aligned}
$$

which implies that

$$
\mu=\frac{7}{3} \frac{E_{M}}{M}
$$

Equation (17) now reads

$$
\frac{1}{2}\left[\frac{3}{4 \pi} \rho_{M}(x)\right]^{2 / 3}+\phi_{f_{M}}(x)=\mu \quad \text { on } \operatorname{supp}\left(\rho_{M}\right)
$$

and the condition $\rho_{M} \geq 0$ is now equivalent to $\phi_{f_{M}} \leq \mu$. Note that according to Lemma $6, \phi_{f_{M}}$ is nondecreasing: as a consequence, $\rho_{M}$ is monotone decreasing (as a radial function) on its support and $\phi_{f_{M}}$ is monotone increasing.

Lemma 13 With the notations of Lemma 12, $\phi_{f_{M}}$ is unique and continuously differentiable. Furthermore, if $f$ is another minimum of $E$ on $\Gamma_{M}$, then there exists $y \in \mathbb{R}^{3}$ such that

$$
\int_{\mathbb{R}^{3}} f(x, v) d v=\rho_{M}(x-y) \quad \text { a.e. } x \in \mathbb{R}^{3} .
$$

Proof. Let us rewrite the Poisson equation for $\phi_{f_{M}}$ using (14) and (17):

$$
\Delta \phi_{f_{M}}=4 \pi \rho_{M}(x)= \begin{cases}\frac{1}{3}(4 \pi)^{2}\left[2\left(\frac{7}{3} \frac{E_{M}}{M}-\phi_{f_{M}}\right)\right]^{3 / 2} & \text { if } \phi_{f_{M}}(x) \leq \frac{7}{3} \frac{E_{M}}{M} \\ 0 & \text { otherwise }\end{cases}
$$

Since $\phi_{f_{M}}$ is radially symmetric, this equation can be rewritten for

$$
w(r)=\frac{7}{3} \frac{E_{M}}{M}-\phi_{f_{M}}(r / \sqrt{c})
$$

as

$$
\left(r^{2} w^{\prime}(r)\right)^{\prime}+r^{2} w_{+}^{3 / 2}(r)=0
$$

with $r=|x|$ and $c=\frac{1}{3} 32 \sqrt{2} \pi^{2}$. Let $R=\frac{3 M^{2}}{7\left|E_{M}\right|} \sqrt{c}$. According to Lemma 6 (see the proof: $\phi_{f_{M}}(r)=-\frac{M}{r}$ for any $\left.r \geq R / \sqrt{c}\right)$,

$$
0=w(R)=\frac{7}{3} \frac{E_{M}}{M}+\frac{M \sqrt{c}}{R} \text { and } \quad w^{\prime}(R)=-\frac{1}{\sqrt{c}} \phi_{f_{M}}^{\prime}\left(\frac{R}{\sqrt{c}}\right)=-\frac{M \sqrt{c}}{R^{2}} .
$$

The uniqueness and the regularity of $w$ follow by standard ODE results.

The expression of non radial minimizers is a consequence of (12), the fact that the associated mass densities minimize (16), the conservation of the $\mathrm{L}^{p}$-norm by radial nonincreasing rearrangements and Riesz' theorem (see Theorem 4 in Appendix A).

This also concludes the proof of Theorem 2. Note that the minimizer with radial symmetric density was previously found as a solution of the VP system in [3], but in a different context. 


\subsection{Nonlinear stability for the evolution problem}

Using the conservation of mass and energy, we obtain on $\Gamma_{M}$ a nonlinear stability result of the minimal energy solution for the evolution problem. We follow the strategy of Guo in [6]. Consider for any $g, h \in \Gamma_{M}$ the distance $d$ defined by

$$
d(g, h)=E(g)-E(h)+\frac{1}{4 \pi}\left\|\nabla \phi_{g}-\nabla \phi_{h}\right\|_{\mathrm{L}^{2}\left(\mathbb{R}^{3}\right)}^{2}
$$

where $\phi_{g}$ and $\phi_{h}$ are solutions of the Poisson equation with mass densities associated to $g$ and $h$ respectively.

Theorem 3 For every $\epsilon>0$, there exists a $\delta>0$ such that the following property holds. If $f$ is a solution of the VP system with an initial condition $f_{0} \in \Gamma_{M}$, then

$$
d\left(f_{0}, f_{M}\right) \leq \delta \quad \Longrightarrow \quad d\left(f^{*}(t), f_{M}\right) \leq \epsilon \quad \forall t \geq 0
$$

Proof. The result is easily achieved by contradiction since $E\left(f^{*}(t)\right)-E\left(f_{M}\right) \leq E\left(f_{0}\right)-$ $E\left(f_{M}\right) \searrow 0$ implies $\left\|\nabla \phi_{f^{*}(t)}-\nabla \phi_{f_{M}}\right\|_{L^{2}\left(\mathbb{R}^{3}\right)} \searrow 0$.

\section{Large time behaviour}

The Galilean invariance of a classical solution $f$ to the VP system with initial data $f_{0}(x, v)$ means that for any $u \in \mathbb{R}^{3}$, the solution with initial data $f_{0}^{u}(x, v)=f_{0}(x, v-u)$ is given by

$$
f^{u}(t, x, v)=f(t, x-t u, v-u) \quad \forall(t, x, v) \in(0,+\infty) \times \mathbb{R}^{3} \times \mathbb{R}^{3} .
$$

\subsection{Galilean invariance and asymptotic behaviour}

The Galilean translations give rise to a family of solutions with same $L^{p}$-norm and potential energy for every $t \geq 0$, parametrized by $u \in \mathbb{R}^{3}$. Nevertheless, other quantities like the total momentum

$$
\langle v\rangle\left(f^{u}\right):=\int_{\mathbb{R}^{6}} v f^{u}(t, x, v) d x d v=\langle v\rangle(f)+u\|f(t)\|_{\mathrm{L}^{1}\left(\mathbb{R}^{6}\right)}
$$

and the total energy

$$
E\left(f^{u}\right)=E(f)+u \cdot\langle v\rangle(f)+\frac{1}{2}|u|^{2}\|f\|_{\mathrm{L}^{1}\left(\mathbb{R}^{6}\right)} .
$$

are not invariant under Galilean translations. Note that

$$
\langle v\rangle^{2}(f) \leq 2\|f\|_{\mathrm{L}^{1}\left(\mathbb{R}^{6}\right)} E_{K I N}(f)
$$

and among the family $\left(f^{u}\right)_{u \in \mathbb{R}^{3}}$, the minimum of $E_{K I N}\left(f^{u}\right)$ is reached by

$$
E_{K I N}\left(f^{\bar{u}}\right)=E_{K I N}(f)-\frac{\langle v\rangle^{2}(f)}{2\|f\|_{\mathrm{L}^{1}\left(\mathbb{R}^{6}\right)}} \quad \text { for } \bar{u}=-\frac{\langle v\rangle(f)}{\|f\|_{\mathrm{L}^{1}\left(\mathbb{R}^{6}\right)}} .
$$

Also note that $u=\bar{u}$ is the unique value of the parameter for which

$$
\langle v\rangle\left(f^{u}\right)=0 .
$$

This can be summarized by the following statement. 
Lemma 14 Let $f \in \mathrm{L}^{1}\left(\mathbb{R}^{6}\right)$ be a distribution function with finite mass and energy. If

$$
E(f)<\frac{1}{2} \frac{\langle v\rangle^{2}(f)}{\|f\|_{\mathrm{L}^{1}\left(\mathbb{R}^{6}\right)}},
$$

then, with the above notations, the function $f^{\bar{u}}$ reaches a negative total energy value. Otherwise, every element of the parametric family of the Galilean translation has nonnegative energy. In any case, the minimal energy solution of the parametric family has null momentum.

Since the quantities involved in (18) are all time independent, the result also holds for any $t$ for the solution $f(t, \cdot, \cdot)$ to the VP system with initial data $f_{0}$.

Proposition 15 Let $f_{0} \in \mathrm{L}^{1} \cap \mathrm{L}^{\infty}\left(\mathbb{R}^{6}\right)$ be a nonnegative distribution function with finite mass and energy and verifying (19). Then there exists three constants $C_{1}, C_{2}$, $C_{3}>0$ such that the solution $f$ of the VP system with initial data $f_{0}$ verifies for any $t \geq 0$

$$
\begin{aligned}
& C_{1} \leq E_{P O T}(f(t, \cdot, \cdot)) \leq C_{2}, \\
& \left\|\rho_{f}(t, \cdot)\right\|_{L^{5 / 3}\left(\mathbb{R}^{3}\right)} \geq C_{3} .
\end{aligned}
$$

Proof. According to Lemma 1, (3),

$$
8 \pi E_{P O T}(f(t)) \leq \mathcal{C}\left\|f_{0}\right\|_{\mathrm{L}^{1}\left(\mathbb{R}^{6}\right)}^{7 / 6}\left\|f_{0}\right\|_{\mathrm{L}^{\infty}\left(\mathbb{R}^{6}\right)}^{1 / 3}\left(2 E_{K I N}\right)^{1 / 2}(f(t))
$$

with the notation $f(t)=f(t, \cdot, \cdot))$, so that, if $E\left(f_{0}\right)<0$, then

$$
0 \geq E\left(f_{0}\right) \geq C E_{P O T}^{2}(f(t))-E_{P O T}(f(t))
$$

with $C=\frac{1}{2}\left(\frac{8 \pi}{\mathcal{C}}\right)^{2}\left\|f_{0}\right\|_{\mathrm{L}^{1}\left(\mathbb{R}^{6}\right)}^{-7 / 3}\left\|f_{0}\right\|_{\mathrm{L}^{\infty}\left(\mathbb{R}^{6}\right)}^{-2 / 3}$. This means that

$$
E_{P O T}(f(t)) \in\left[\frac{1}{2 C}\left(1-\sqrt{1+4 E\left(f_{0}\right) C}\right), \frac{1}{2 C}\left(1+\sqrt{1+4 E\left(f_{0}\right) C}\right)\right] .
$$

Estimate (20) holds because there exists a function in the family of the Galilean translations associated to $f_{0}$ with negative total energy: it is therefore not restrictive to take $E\left(f_{0}\right)<0$ to evaluate $E_{P O T}(f(t))$. On the other hand (21) is a direct consequence of the Hardy-Littlewood-Sobolev inequalities (see the proof of Lemma 1) with $C_{3}=\frac{8 \pi C_{1}}{\sqrt{\Sigma}}$.

\subsection{Variance and dispersion estimates}

The solutions to the VP system in the gravitational case have a qualitative behaviour which strongly differs from the behaviour in the plasma physics case since, for instance, stationary solutions exist. The rest of this section is devoted to solutions in the gravitational case for which Condition (19) is violated. Our goal is to prove some dispersion estimates. For that purpose, consider the dispersion operators in space and in velocity defined by

$$
<(\Delta x)^{2}>\quad:=\int_{\mathbb{R}^{6}}|x|^{2} f(t, x, v) d x d v-\left(\int_{\mathbb{R}^{6}} x f(t, x, v) d x d v\right)^{2},
$$

and

$$
<(\Delta v)^{2}>:=\int_{\mathbb{R}^{6}}|v|^{2} f(t, x, v) d x d v-\left(\int_{\mathbb{R}^{6}} v f(t, x, v) d x d v\right)^{2} .
$$

Up to a mass normalization, the dispersion operator in space coincides with the statistical variance of the density mass function and, consequently, it is a measure of the 
dispersion of such a distribution. If $f$ is a solution of the VP system, the time evolution of both quantities are related with the total energy and the momentum by the dispersion equation. Since this property is also valid for the VP system in plasma physics we will consider both situations.

Lemma 16 Let $f$ be a classical solution of VP with finite mass, energy and space dispersion. Then, it verifies

$$
\frac{1}{2} \frac{d^{2}}{d t^{2}}<(\Delta x)^{2}>=E(f)+\frac{1}{2}<(\Delta v)^{2}>-\frac{1}{2}\langle v\rangle^{2}(f) .
$$

Proof. A straightforward calculation using the VP system gives

$$
\begin{aligned}
\frac{1}{2} \frac{d^{2}}{d t^{2}} \int_{\mathbb{R}^{6}}|x|^{2} f(t, x, v) d x d v & =\frac{1}{2} \frac{d}{d t} \int_{\mathbb{R}^{6}}|x|^{2}\left(-v \cdot \nabla_{x} f+\nabla_{x} \phi \cdot \nabla_{v} f\right) d x d v \\
& =\int_{\mathbb{R}^{6}}(v \cdot x)\left(-v \cdot \nabla_{x} f+\nabla_{x} \phi \cdot \nabla_{v} f\right) d x d v \\
& =\int_{\mathbb{R}^{6}}|v|^{2} f d x d v-\frac{1}{4 \gamma \pi} \int_{\mathbb{R}^{3}}\left(x \cdot \nabla_{x} \phi\right) \Delta \phi d x \\
& =\int_{\mathbb{R}^{6}}|v|^{2} f d x d v-\frac{1}{8 \gamma \pi} \int_{\mathbb{R}^{3}}|\nabla \phi|^{2} d x \\
& =E(f)+\frac{1}{2} \int_{\mathbb{R}^{6}}|v|^{2} f d x d v
\end{aligned}
$$

which is equivalent to (22).

Equation (22) is equivalent to a formula proposed by R. Illner and G. Rein in [9], and B. Perthame in [15]. As a straightforward consequence, the following pseudo-conformal law holds.

Lemma $17[9,15]$ Let $f_{0} \in \mathrm{L}^{1} \cap \mathrm{L}^{\infty}\left(\mathbb{R}^{6}\right)$ be a nonnegative initial data with finite mass, energy and space dispersion. Then a classical solution $f$ to the VP system with initial data $f_{0}$ satisfies the following identity:

$$
\frac{d}{d t}\left(\int_{\mathbb{R}^{6}}|x-t v|^{2} f(t, x, v) d x d v-\frac{t^{2}}{4 \pi \gamma} \int_{\mathbb{R}^{3}}|\nabla \phi|^{2} d x\right)=-\frac{t}{4 \pi \gamma} \int_{\mathbb{R}^{3}}|\nabla \phi|^{2} d x .
$$

Proof. For completion, let us give a proof of this identity.

$$
\int_{\mathbb{R}^{6}}|x-t v|^{2} f d x d v=\int_{\mathbb{R}^{6}}|x|^{2} f d x d v+t^{2} \int_{\mathbb{R}^{6}}|v|^{2} f d x d v-t \frac{d}{d t}\left(\int_{\mathbb{R}^{6}}|x|^{2} f d x d v\right)
$$

Then, the left hand side term of (24) can be written as

$$
\begin{array}{r}
\frac{d}{d t}\left[\int_{\mathbb{R}^{6}}|x|^{2} f d x d v-t \frac{d}{d t}\left(\int_{\mathbb{R}^{6}}|x|^{2} f d x d v\right)+t^{2} \int_{\mathbb{R}^{6}}|v|^{2} f d x d v-\frac{t^{2}}{4 \pi \gamma} \int_{\mathbb{R}^{3}}|\nabla \phi|^{2} d x\right] \\
=\frac{d}{d t}\left[\int_{\mathbb{R}^{6}}|x|^{2} f d x d v-t \frac{d}{d t}\left(\int_{\mathbb{R}^{6}}|x|^{2} f d x d v\right)+2 t^{2} E(f)\right] \\
=-t \frac{d^{2}}{d t^{2}} \int_{\mathbb{R}^{6}}|x|^{2} f d x d v+4 t E(f)=-\frac{t}{4 \pi \gamma} \int_{\mathbb{R}^{3}}|\nabla \phi|^{2} d x
\end{array}
$$

where in the last equality we have used (23).

Consider now the solutions with positive energy, in the case $\gamma=+1$.

Proposition 18 Let $f$ be a solution of the VP system in the gravitational case with positive energy corresponding to a nonnegative initial datum $f_{0} \in \mathrm{L}^{1}\left(\mathbb{R}^{6}\right) \cap \mathrm{L}^{\infty}\left(\mathbb{R}^{6}\right)$ 
with finite mass, energy and space dispersion. Then, there exists positive constants $C$, $C_{1}, C_{2}$ such that for some $t_{0}>0$,

$$
C_{1} t^{2} \leq \int_{\mathbb{R}^{6}}|x|^{2} f(t, x, v) d x d v \leq C_{2} t^{2} \quad \forall t \geq t_{0}>0,
$$

and, for any $p \in[1, \infty)$,

$$
\|\rho(t, x)\|_{\mathrm{L}^{p}\left(\mathbb{R}^{3}\right)} \geq \frac{C}{t^{3(p-1) / p}}, \quad \forall t>t_{0},
$$

where $C_{i}$ depend on $E(f),\left\|f_{0}\right\|_{\mathrm{L}^{1}\left(\mathbb{R}^{6}\right)},\left\|f_{0}\right\|_{\mathrm{L}^{\infty}\left(\mathbb{R}^{6}\right)}$ and $C$ also depends on $p$.

Proof. We can rewrite (23) as

$$
\frac{1}{2} \frac{d^{2}}{d t^{2}}\left(\int_{\mathbb{R}^{6}}|x|^{2} f(t, x, v) d x d v\right)=2 E(f)+E_{P O T}(f) .
$$

Combining this and the estimate of Theorem 1, we find

$$
2 E(f) \leq \frac{1}{2} \frac{d^{2}}{d t^{2}} \int_{\mathbb{R}^{6}}|x|^{2} f(t, x, v) d x d v \leq 2 E(f)+C,
$$

where $C$ depends on $E(f),\left\|f_{0}\right\|_{\mathrm{L}^{1}\left(\mathbb{R}^{6}\right)},\left\|f_{0}\right\|_{\mathrm{L}^{\infty}\left(\mathbb{R}^{6}\right)}$. This estimate proves (25) by integrating twice in time. As for the estimate on $\rho$, we may write

$$
\begin{aligned}
\int_{\mathbb{R}^{6}} f(t, x, v) d x d v & \leq \int_{\mathbb{R}^{3}} \int_{|x| \leq R} f(t, x, v) d x d v+\int_{\mathbb{R}^{3}} \int_{|x|>R} f(t, x, v) d x d v \\
& \leq\left(\frac{4 \pi}{3} R^{3}\right)^{(p-1) / p}\|\rho(t, \cdot)\|_{L^{p}\left(\mathbb{R}^{3}\right)}+\frac{1}{R^{2}} \int_{\mathbb{R}^{6}}|x|^{2} f(t, x, v) d x d v \\
& \leq C\|\rho(t, x)\|_{\mathrm{L}^{p}\left(\mathbb{R}^{3}\right)}^{\frac{2 p}{5 p-3}}\left(\int_{\mathbb{R}^{6}}|x|^{2} f(t, x, v) d x d v\right)^{\frac{3 p-3}{5 p-3}}
\end{aligned}
$$

where in the last line we optimized on $R>0$. The conclusion holds because of the time preservation of the $\mathrm{L}^{1}\left(\mathbb{R}^{6}\right)$-norm and Estimate (25).

This argument can be used for solutions of the VP system in the plasma physical case and provides the same type of results: see [5] (with a different approach). Observe furthermore that Proposition 18 does not imply any dispersion property in the usual sense, as can be shown by considering a Galilean translation of a stationary solution with positive energy (i.e. for $|u|$ big enough). This motivates the last result of the paper.

Proposition 19 Let $f$ be a solution of the VP system in the gravitational case with positive energy corresponding to a nonnegative initial datum $f_{0} \in \mathrm{L}^{1}\left(\mathbb{R}^{6}\right) \cap \mathrm{L}^{\infty}\left(\mathbb{R}^{6}\right)$ with mass $\left\|f_{0}\right\|_{\mathrm{L}^{1}\left(\mathbb{R}^{6}\right)}=1$, and finite energy and space dispersion. Assume that

$$
E\left(f_{0}\right)>\frac{1}{2}\left|\int_{\mathbb{R}^{6}} v f_{0}(x, v) d x d v\right|^{2} .
$$

Then, there exists a $t_{0}>0$ and two positive constants $C_{1}, C_{2}$ such that

$$
C_{1} t^{2} \leq<(\Delta x)^{2}>\leq C_{2} t^{2} \quad \forall t \geq t_{0},
$$

where $C_{i}$ depend on $E(f),\left\|f_{0}\right\|_{\mathrm{L}^{1}\left(\mathbb{R}^{6}\right)}$ and $\left\|f_{0}\right\|_{\mathrm{L}^{\infty}\left(\mathbb{R}^{6}\right)}$.

Proof. The space dispersion operator is invariant under Galilean translations

$$
<(\Delta x)^{2}>\left(f^{u}\right) \quad=\quad<(\Delta x)^{2}>(f) \quad \forall u \in \mathbb{R}^{3} .
$$


With the notations of the beginning of this section, consider the Galilean translation of $f$ with minimal energy and null momentum. The dispersion equation (22) applied to this function $f^{\bar{u}}$ reads as

$$
\frac{1}{2} \frac{d^{2}}{d t^{2}}<(\Delta x)^{2}>=E\left(f^{\bar{u}}\right)+\frac{1}{2}<(\Delta v)^{2}>
$$

where

$$
E\left(f^{\bar{u}}\right)=E\left(f_{0}\right)-\frac{1}{2}|\bar{u}|^{2}, \quad \bar{u}=\langle v\rangle\left(f_{0}\right)=-\int_{\mathbb{R}^{6}} v f_{0}(x, v) d x d v,
$$

so that $E\left(f^{\bar{u}}\right)$ is positive by $(26)$. Since $<(\Delta v)^{2}>$ is positive and bounded by Lemma 3 , we deduce

$$
E(f)<\frac{1}{2} \frac{d^{2}}{d t^{2}}<(\Delta x)^{2}><E(f)+C,
$$

where $C$ is controlled in terms of $E(f)$ by Theorem 1 . This ends the proof by integrating twice in time.

\section{Appendix A - Symmetric nonincreasing rearrange- ments}

This appendix is devoted to the statement of basic properties of symmetric nonincreasing rearrangements of nonnegative functions. Such a tool has been widely used in open quantum problems (see for example $[17,12])$. As a special case, we consider functions of the variables $x$ and $v$, which are rearranged with respect to the $x$ variable only (see [11]).

The symmetric rearrangement $A^{*}$ of the set $A$ in $\mathbb{R}^{n}, n \geq 1$, is the open ball in $\mathbb{R}^{n}$ centered at the origin whose volume is that of $A$. The symmetric nonincreasing rearrangement of the characteristic function $\chi_{A}$ of $A$ is then defined by

$$
\chi_{A}^{*}:=\chi_{A^{*}}= \begin{cases}1 & \text { if } \frac{1}{n}\left|S^{n-1}\right||x|^{n} \leq\left\|\chi_{A}\right\|_{\mathrm{L}^{1}\left(\mathbb{R}^{n}\right)} \\ 0 & \text { otherwise. }\end{cases}
$$

Let $h: \mathbb{R}^{n} \rightarrow \mathbb{C}$ be a Borel measurable function such that $\left\|\chi_{\{|h|>t\}}\right\|_{L^{1}\left(\mathbb{R}^{\mathrm{n}}\right)}$ is finite for all $t$. Here we denote by $\{|h|>t\}$ the set $\left\{x \in \mathbb{R}^{n}:|h(x)|>t\right\}$. Then

$$
|h(x)|=\int_{0}^{\infty} \chi_{\{|h|>t\}}(x) d t
$$

holds and we can define the nonincreasing rearrangement of $h$ by

$$
h^{*}(x):=\int_{0}^{\infty} \chi_{\{|h|>t\}}^{*}(x) d t .
$$

The symmetric nonincreasing rearrangement of a function $(x, v) \mapsto g(x, v) \geq 0$ with respect to the $\mathrm{x}$ variable only (i.e. for fixed $v$ ) is then defined as

$$
g^{*_{x}}(x, v):=\int_{0}^{\infty} \chi_{\left\{x \in \mathbb{R}^{n}: g(x, v)>t\right\}}^{*} d t .
$$

Thanks to the Fubini's theorem we can easily adapt to the case of the symmetric nonincreasing rearrangement with fixed $v$ the standard properties of the usual symmetric 
nonincreasing rearrangements:

$$
\begin{aligned}
\int_{\mathbb{R}^{2 n}} g^{*_{x}}(x, v) d x d v & =\int_{\mathbb{R}^{2 n}} g(x, v) d x d v, \\
\left\|g^{* x}\right\|_{L^{\infty}\left(\mathbb{R}^{2 n}\right)} & =\|g\|_{L^{\infty}\left(\mathbb{R}^{2 n}\right)}, \\
\int_{\mathbb{R}^{2 n}}|v|^{2} g^{*_{x}}(x, v) d x d v & =\int_{\mathbb{R}^{2 n}}|v|^{2} g(x, v) d x d v, \\
\int_{\mathbb{R}^{n}} g^{*_{x}}(x, v) d v & =\int_{\mathbb{R}^{n}} g\left(x^{\prime}, v\right) d v \quad \text { if }|x|=\left|x^{\prime}\right|, \\
\int_{\mathbb{R}^{n}} g^{*_{x}}(|x|, v) d v & \geq \int_{\mathbb{R}^{n}} g^{* x}(|y|, v) d v \quad \text { if }|x| \leq|y|, \\
\int_{\mathbb{R}^{n}} \psi(|x|) g^{*_{x}}(|x|, v) d v & \leq \int_{\mathbb{R}^{n}} \psi(|x|) g(x, v) d v,
\end{aligned}
$$

where in the last inequality the function $r \mapsto \psi(r)$ is nondecreasing. If moreover $\psi$ is (strictly) increasing on $\mathbb{R}^{+}$, then the inequality in (32) is strict almost everywhere in $v \in \mathbb{R}^{n}$ unless $g^{*_{x}} \equiv g$ almost everywhere on $\mathbb{R}^{n} \times \mathbb{R}^{n}$.

For completion, let us state Riesz' theorem (see [11]):

Theorem 4 Let $f, g$ and $h$ be three nonnegative functions on $\mathbb{R}^{n}$. Then

$$
\int_{\mathbb{R}^{n}} \int_{\mathbb{R}^{n}} f(x) g(x-y) h(y) d x d y=: I(f, g, h) \leq I\left(f^{*}, g^{*}, h^{*}\right),
$$

with the convention that $I\left(f^{*}, g^{*}, h^{*}\right)=\infty$ if $I(f, g, h)=\infty$. If $g$ is radially symmetric and strictly decreasing, i.e. if $g(x)>g(y)$ for any $x$, $y$ such that $|x|<|y|$, equality in (33) holds only if $f(x)=f^{*}(x-y)$ and $h(x)=h^{*}(x-y)$ for some $y \in \mathbb{R}^{n}$.

\section{Appendix B - Explicit form of the optimal constant}

Let $w$ be the solution of the ODE

$$
\left\{\begin{array}{l}
\left(r^{2} w^{\prime}\right)^{\prime}+r^{2} w_{+}^{3 / 2}=0 \quad r \in[0,+\infty) \\
w(0)=1, \quad w^{\prime}(0)=0
\end{array}\right.
$$

Note that $w^{\prime} \leq 0$ as long as $w \geq 0$. Let $u$ be given by $w(r)=u(-\log r) / r^{4}$. Then it solves the equation: $u^{\prime \prime}+7 u^{\prime}+12 u+u_{+}^{3 / 2}=0$. A phase diagram analysis of $\left(u, u^{\prime}\right)$, shows that $w$ has to change sign. Denote by $\sigma$ its first positive zero and define the quantities

$$
\begin{aligned}
& A:=\int_{0}^{\sigma} r^{2} w^{5 / 2} d r, \\
& B:=\int_{0}^{\sigma} r^{2} w^{3 / 2} d r .
\end{aligned}
$$

The best constant $\mathcal{C}$ in Inequality (3) of Lemma 1 is defined as

$$
\mathcal{C}^{-1}=\inf \left[\|f\|_{L^{1}\left(\mathbb{R}^{6}\right)}^{7 / 6}\|f\|_{L^{\infty}\left(\mathbb{R}^{6}\right)}^{1 / 3} \frac{\left(\int_{\mathbb{R}^{6}}|v|^{2} f(x, v) d x d v\right)^{1 / 2}}{\int_{\mathbb{R}^{3}}|\nabla \phi|^{2} d x}\right]
$$

where the infimum is taken over the set of the functions $f \in \mathrm{L}^{1} \cap \mathrm{L}^{\infty}\left(\mathbb{R}^{6}\right)$ such that $f \geq 0,|v|^{2} f \in \mathrm{L}^{1}\left(\mathbb{R}^{6}\right), f \not \equiv 0$. 
Proposition 20 With the above notations,

$$
\mathcal{C}=8 \pi M^{-7 / 6}\left(2 J_{M}\right)^{-1 / 2}=8 \pi \frac{\left(2\left|E_{M}\right|\right)^{1 / 2}}{M^{7 / 6}}
$$

is independent of $M>0$ and takes the value:

$$
\mathcal{C}=32 \pi^{2} \sqrt{\frac{2^{5 / 2} a^{7 / 4} A}{5 c^{3 / 2}}}
$$

where $a=\frac{c^{2}}{4}\left(\frac{3}{(4 \pi)^{2} B}\right)^{4 / 3}$ and $c=\frac{1}{3} 32 \sqrt{2} \pi^{2}$.

Proof. The independence in $M$ is a consequence of the scaling invariance (see Lemma 7) and the fact that according to Lemma $2, \mathcal{C}$ is achieved by the minima of the functional $E$ on $\Gamma_{M}$. Without restriction, we can assume that $M=1$. Let $f=f_{1}, \rho=\rho_{1}$ and $\phi=\phi_{f_{1}}$ be the corresponding mass density and potential. From Theorem 2, we get

$$
\Delta \phi=4 \pi \rho=\frac{1}{3}(4 \pi)^{2}\left[2\left(-\frac{7}{6}\left(\frac{\mathcal{C}}{8 \pi}\right)^{2}-\phi\right)_{+}\right]^{3 / 2} .
$$

On the other hand, by the proof of Lemma 11 and Corollary 3, we get

$$
\int_{\mathbb{R}^{6}}|v|^{2} f d x d v=\frac{4 \pi}{5} \int_{\mathbb{R}^{3}}\left(\frac{3}{4 \pi} \rho\right)^{5 / 3} d x=\frac{1}{8 \pi} \int_{\mathbb{R}^{3}}|\nabla \phi|^{2} d x=2\left|E_{M}\right| .
$$

Thus we obtain

$$
\mathcal{C}=\sqrt{8 \pi}\left(\int_{\mathbb{R}^{3}}|\nabla \phi|^{2} d x\right)^{1 / 2}
$$

With the notations of the proof of Lemma $13, w_{a}(r)=-\frac{7}{6}\left(\frac{\mathcal{C}}{8 \pi}\right)^{2}-\phi_{f_{M}}(r / \sqrt{c})$ is a solution of

$$
\left\{\begin{array}{l}
\left(r^{2} w_{a}^{\prime}(r)\right)^{\prime}+r^{2}\left(w_{a}\right)_{+}^{3 / 2}(r)=0 \\
w_{a}(0)=a, \quad w_{a}^{\prime}(0)=0
\end{array}\right.
$$

where $a>0$ has to be determined in order that

$$
1=\int_{\mathbb{R}^{3}} \rho d x=\frac{1}{3}(4 \pi)^{2} \int_{0}^{\sigma(a) / \sqrt{c}} r^{2}\left(2 w_{a}(\sqrt{c} r)\right)^{3 / 2} d r .
$$

Here $\sigma(a)$ denotes the smallest zero of $w_{a}$. Note that $\rho(r)=\frac{1}{3} 4 \pi\left[2 w_{a}(\sqrt{c} r)\right]^{3 / 2}$ for $r \leq \sigma(a) / \sqrt{c}$. The scaling invariance

$$
w_{a}(r)=a w_{1}\left(a^{1 / 4} r\right)
$$

reduces the computation to the case $a=1, w=w_{1}$ given by $(34)$ : on $(0, \sigma(a) / \sqrt{c})$, $\rho(r)=\frac{1}{3} 4 \pi\left[2 a w\left(a^{1 / 4} \sqrt{c} r\right)\right]^{3 / 2}$, so that

$$
1=\frac{1}{3}(4 \pi)^{2}\left(\frac{2}{c}\right)^{3 / 2} a^{3 / 4} \int_{0}^{\sigma} r^{2} w^{3 / 2} d r=\frac{1}{3}(4 \pi)^{2}\left(\frac{2}{c}\right)^{3 / 2} a^{3 / 4} B,
$$

where $\sigma=\sigma(1)$, and allows to express $a$ in terms of $B$ :

$$
a=\frac{c^{2}}{4}\left(\frac{3}{(4 \pi)^{2} B}\right)^{4 / 3} .
$$

Similarly, we compute

$$
\int_{\mathbb{R}^{3}}|\nabla \phi|^{2} d x=\frac{1}{5} 32 \pi^{2} \int_{\mathbb{R}^{3}}\left(\frac{3}{4 \pi} \rho\right)^{5 / 3} d x=\frac{1}{5} 32 \pi^{2} 4 \pi 2^{5 / 2} a^{7 / 4} c^{-3 / 2} A .
$$


This gives the expression of $\mathcal{C}$ simply by collecting the estimates.

Remark The expression given in Proposition 20 is not easy to use. A numerical computation provides $\mathcal{C} \approx 54.62 \ldots$. Going back to Lemma 1 , we may wonder if the estimate given in the proof is optimal. This is actually not the case. Let $\Sigma$ be the optimal constant in the Hardy-Littlewood-Sobolev inequality

$$
\int_{\mathbb{R}^{3}}|\nabla \phi|^{2} d x=4 \pi \int_{\mathbb{R}^{3}} \frac{\rho(x) \rho(y)}{|x-y|} d x d y \leq 4 \pi \Sigma\|\rho\|_{L^{6 / 5}\left(\mathbb{R}^{3}\right)}^{2},
$$

which, according to [13], is $\Sigma=\frac{4}{3}\left(\frac{4}{\sqrt{\pi}}\right)^{2 / 3}$. Keeping track of the constants in the interpolation identity, we get the following estimate :

$$
\mathcal{C} \leq 4 \pi \Sigma\left(\frac{5}{3}(2 \pi)^{2 / 5}\right)^{5 / 6}=\frac{16 \pi}{3}\left(\frac{20}{3}\right)^{5 / 6} \approx 81.42 \ldots
$$

Acknowledgments. This research was partially supported by the EU financed network IHP-HPRN-CT-2002-00282 and by MCYT (Spain), Proyecto BFM2002-00831.

\section{References}

[1] C. Bardos, P. Degond, Global existence for the Vlasov-Poisson equation in 3 space variables with small initial data, Ann. Inst. H. Poincaré, Analyse Non Linéaire 2 no. 2 (1985), 101-118.

[2] J. BATT, Asymptotic properties of spherically symmetric self-gravitating mass systems for $t \rightarrow \infty$, Transport Theory and Statist. Phys. 16 no. 4-6, (1987), 763-778.

[3] J. BAtT, W. FAltenBACher, E. Horst, Stationary spherically symmetric models in stellar dynamics, Arch. Rational Mech. Anal. 93 no. 2 (1986), 159-183.

[4] J. BATT, K. PFAfFelmoser, On the radius continuity of the models of polytropic gas spheres which correspond to the positive solutions of the generalized EmdemFowler equation, Math. Methods Appl. Sci. 10 no. 5 (1988), 499-516.

[5] F. Castella, Propagation of space moments in the Vlasov-Poisson equation and further results, Ann. Inst. H. Poincaré Anal. Non Linéaire 16 no. 4 (1999), 503533.

[6] Y. Guo, Variational method for stable polytropic galaxies, Arch. Ration. Mech. Anal. 150 no. 3 (1999), 209-224.

[7] Y. Guo, G. Rein, Stable steady states in stellar Dynamics, Arch. Ration. Mech. Anal. 147 no. 3 (1999), 225-243.

[8] E. Horst, On the classical solutions of the initial value problem for the unmodified non-linear Vlasov equation. I. General Theory, Math. Methods Appl. Sci. 3 (1981), 229-248, and II. Special Cases, Math. Methods Appl. Sci. 4 (1982), 19-32.

[9] R. Illner, G. Rein, Time decay of the solutions of the Vlasov-Poisson system in the plasma physical case, Math. Methods Appl. Sci. 19 no. 17 (1996), 1409-1413.

[10] R. KuRTh, A global particular solution to the initial value problem of stellar dynamics, Quart. Appl. Math. 36 (1978), 325-329.

[11] E. Lieb, M. Loss, Analysis, American Mathematical Society, 1997.

[12] E. H. LIEB, Existence an uniqueness of the mininimizing solution of Choquard's Nonlinear Equation, Studies in Appl. Math. 57 no. 2 (1977), 93-105.

[13] E. H. LIEB, Sharp constants in the Hardy-Littlewood-Sobolev and related inequalities, Annals of Mathematics 118 (1983), 349-374. 
[14] P.-L. Lions, The concentration-compactness principle in the calculus of variations. Part I. The locally compact case, Ann. Inst. H. Poincaré 1 (1984), 109-145.

[15] B. Perthame, Time decay, propagation of low moments and dispersive effects for kinetic equations, Comm. Partial Differential Equations 21 no. 3-4 (1996), 659-686.

[16] G. REIN, Reduction and a concentration-compactness principle for EnergyCasimir functionals, Siam J. Math. Anal. 33 (2001), 896-912.

[17] E. Ruiz Arriola, J. Soler, A Variational approach to the Schrödinger-Poisson system: asymptotic behaviour, breathers and stability, J. Stat. Phys. 103 no. 5-6 (2001), 1069-1106. 